that it is not the content of the crop which determines whether a fly shows hunger or not.

Marchal $^{2}$ claims that a Dytiscus in which the frontal ganglion is destroyed is unable to swallow. In nine cases where the ganglion was removed from flies, they were able to drink and swallow, and in none of the cases did the operation appear to influence the hunger-reaction. The peristaltic movements were not obviously disturbed. A similar result was obtained from a control experiment with a grasshopper from which the left half of the head and thorax was removed. It swallowed normally after the removal of the frontal ganglion. I am thus unable to explain the function of this ganglion.

I tried to sever the sympathetic nerve connexion from the mid-intestine to the posterior side of the brain. Three flies in which the oesophagus was ligatured behind the corpus cardiacum + ganglion hypocerebrale and the passage to the crop, just in front of the proventriculus ${ }^{1}$, regained their hunger-reaction in its full strength. They drank more sugar water than normal flies and seemed unable to satisfy their hunger. Dissection afterwards showed that the midintestine contained sugar water. Inhibiting impulses seem thus to be conducted from the mid-intestine to the posterior part of the brain soon after a small amount of sugar water has passed through the proventriculus. The hunger-condition, however, is maintained for some time after a meal. This becomes evident from the fact that newly fed flies still turned towards a droplet of sugar water when touching it with their front tarsi, but, however, without drinking. After some time this reaction ceased. Preliminary experiments seem to indicate that the actual hungerreaction is caused by the effect of the exhausted hæmolymph on the central nervous system.

The work is still in progress and further details will later be published elsewhere.

Zoology Department,

University of the Witwatersrand, Johannesburg. Aug. 20.

${ }^{1}$ Thomsen, E., Videnskab. Medd. Dansk Naturh. Forening, 108 (1942). S See Wigglesworth, V. B., "The Principles of Insect Physiology"
$(19+2)$.

\section{Breeding of the Edible Turtle}

THe scanty literature on the green or edible turtle, Chelonia mydas, as reviewed by Ingle and Smith ${ }^{1}$, agrees in allocating a definite breeding season (in no case more than six months) to this species, varying from April-August in the West Indies to OctoberFebruary in Queensland.

The significance of monthly figures -from three islands off the Sarawak (Borneo) coast-summarized by Banks ${ }^{2}$ appears to have been overlooked, perhaps because they were derived from Malay collectors and contain some noticeable contradictions (for example, totals on pp. 527 and 530 of ref. 2). Since 1947, accurate figures have been kept from these three islands, now no longer controlled by the Malay chieftains but through the Curator, Sarawak Museum There can be no question that green turtles do breed in every month of the year on each island; a perm. anent staff collects eggs nightly. Adults are never killed. Figures for 1950 (including eggs replanted for hatching) are reasonably typical. The 1950 total of $2,357,644$ eggs recorded was distributed as in the accompanying table.

\begin{tabular}{lclc} 
Month & No, of eggs & \multicolumn{1}{c}{ Month } & No. of eggs \\
January & 24,264 & July & 495,191 \\
February & 26,205 & August & $\mathbf{5 0 3 , 6 8 8}$ \\
Marrh & 42,798 & September & 370,626 \\
April & 75,247 & October & 194,228 \\
May & 171,510 & November & 90,122 \\
June & 315,533 & December & $\mathbf{4 8 , 2 3 2}$
\end{tabular}

Despite a definite 'summer' peak, 12 per cent of the eggs were laid in the six full monsoon months (November-April), which in 1950 was often severe, making it very difficult for the turtles to get ashore on the three tiny (total six acres) beaches. On only one night (December 12) in the year did no turtle come ashore and lay.

This situation raises points of general interest in connexion with breeding cycles, and is being further studied. It is, however, possible that it is not peculiar to this area. Careful studies of this economically important and readily observed species are exceedingly meagre. Even the fullest study, that of Moorhouse ${ }^{3}$ on the Great Barrier Reef, only covers five months (1929-30) and takes much for granted. For example, referring to statements by previous writers that incubation takes six to eight weeks, he concludes (ref. 3, p. 10) that "the present investigations have proved this a fallacy"- - because his observations gave 65-72 days. But he only studied eleven nests. In Sarawak, the longest incubation period recorded so far is 65 days, the shortest 50 . Ninety clutches laid in the height of the 1950 season (August) averaged 52 days.

In the monsoon, hatching is on the average slower. There are interesting possibilities for comparative work over the enormous range of this common (though decreasing) species, the habits of which lend themselves to exact observation and statistical checking. We here would welcome any research co-operation or co-ordination.

\section{TOM HARRISSON} (Curator)

The Museum, Kuching, Sarawak. Aug. 30.

${ }^{1}$ Ingle, Robert M., and Smith, F. G. Walton, "Sea Turtles and the Turtle Industry of the West Indies, Florida, and the Gulf of Mexico ; with Annotated Bibliography" (Univ. Miami Pub., 1948).

' Banks, E., Sarawak Mus. J., 4, No. 15 (1937).

"Moorhouse, F. W. "Reports of the Great Barrier Reef Committee", 4, Pt. 1 (1933).

\section{Similar Joint Action of Insecticides}

BuIss ${ }^{1}$ defined the similar joint action of poisons in such a way that the quantal responses to mixtures of poisons acting similarly could be predicted from the separate toxicities of the components of the mixtures. Finney ${ }^{2}$ revised Bliss's treatment of the subject, and both Finney ${ }^{2}$ and Swisher ${ }^{3}$ have shown that mixtures of certain insecticides were of the toxicity so predicted. If $z_{3}$ and $z_{2}$ are the doses of poisons $A$ and $B$ respectively, the probit responses, $y$, produced by $A$ and $B$ alone are usually given respectively by :

$$
\begin{aligned}
& y=\alpha_{1}+\beta_{1} \log z_{1}, \\
& y=\alpha_{2}+\beta_{2} \log z_{2} .
\end{aligned}
$$

Provided, and only provided, that $\beta_{1}=\beta_{2}=\beta$, the probit response to $z_{1}$ of $A$ and $z_{2}$ of $B$ applied together is, according to Bliss's scheme, predicted by Finney's equation (8.4), which may be conveniently expressed in the form:

$$
y=\beta \log \left(z_{1} h^{a_{1} / \beta}+z_{2} h^{a_{2} / \beta}\right),
$$

